\title{
Hypoxia-responsive miR-346 promotes proliferation, migration, and invasion of renal cell carcinoma cells via targeting NDRG2
}

\author{
Z. H. SU, H. H. LIAO, K. E. LU, Z. CHI, Z. Q. QIU, J. M. JIANG, Z. WU* \\ Department of Urology, Affiliated Hospital of Guangdong Medical University, Zhanjiang, China \\ *Correspondence: jiaoxie58478433845@126.com
}

Received September 17, 2019 / Accepted January 7, 2020

\begin{abstract}
Renal cell carcinoma (RCC) is the most common malignant tumor of the kidney. In this study, we investigated the role of miR-346 in RCC cells under hypoxia. OS-RC-2 and 786-O cells were cultured in 1\% O2 or normal oxygen. Cell proliferation, migration, and invasion abilities were measured by 3-(4,5-dimethylthiazol-2-yl)-2,5-diphenyl tetrazolium bromide (MTT) assay, transwell migration, and invasion assays, respectively. Quantitative real-time PCR (qRT-PCR) was performed to detect the expression of miR-346 and N-myc downstream-regulated gene 2 (NDRG2). Then bioinformatics analysis, dual-luciferase reporter assay, and RNA immunoprecipitation were carried out to determine the relationship between miR-346 and NDRG2. The protein expression of NDRG2 was detected by western blot assay. Hypoxia promoted cell proliferation, migration, and invasion in OS-RC-2 and 786-O cells. Meanwhile, we found that miR-346 was upregulated in RCC cells under hypoxia as relative to normoxia. miR-346 deletion could decrease the viability, migration, and invasion abilities of RCC cells under hypoxia. Besides, our data demonstrated that NDRG2 was a target gene of miR-346. The expression of NDRG2 in OS-RC-2 and 786-O cells was lower under hypoxia than under normal oxygen conditions. Moreover, NDRG2 overexpression could inhibit cell proliferation, migration, and invasion in RCC cells under hypoxia. And NDRG2 silencing reversed the inhibitory effects of the miR-346 inhibitor on the viability, migration, and invasion abilities of RCC cells in hypoxia conditions. miR-346 promoted the viability, migration, and invasion of RCC cells under hypoxia by targeting NDRG2.
\end{abstract}

Key words: renal cell carcinoma, hypoxia, miR-346, NDRG2, viability, migration, invasion

Renal cell carcinoma (RCC) is a common malignant tumor among adults and represents about $90 \%$ of all kidney tumors. In the past 20 years, the annual incidence of RCC in worldwide has remarkably increased by about 2-4\% [1]. Of note, it has been reported that the 5-year survival rate was only $12 \%$ when patients were diagnosed at an advanced stage with metastatic RCC, and even lower than $10 \%$ in $13-30 \%$ RCC patients who experienced metastatic recurrence [2-4]. Despite there are multimodal therapeutic methods, the prognosis of metastatic RCC patients is still poor. Accumulating studies indicated that hypoxia is a characteristic feature that contributes to the processes of malignant tumors, which is nearly related to unfavorable prognosis in RCC [5, 6]. Von Hippel-Lindau (VHL) is a suppressor gene related to inherited VHL syndrome, and the inactivating mutations of VHL are characteristic of the human kidney malignancy [7]. Hypoxia-inducible factor (HIF)-1 $\alpha$ and HIF-2 $\alpha$ were stably expressed in hypoxia, or in the case of von Hippel-Lindau (VHL) inactivating mutations, which could be ubiquitinated and degraded by VHL in normoxia [8]. Importantly, HIF-1a and HIF-2 $\alpha$ are regulators in tumor progression. VHL was involved in various types of tumors through the regulation of microRNAs (miRNAs). miRNAs may be biomarkers for early diagnosis of RCC, and hypoxia could evoke the ectopic expression of miRNAs. Therefore, we aimed to investigate the role of miRNAs related to RCC, which might represent a potential therapeutic target.

miRNAs, with the length of about 19-25 nucleotides, are a group of highly conserved small non-protein coding RNAs, which can cause the target gene expression degradation or translational repression [9]. miRNAs play vital roles in various tumor types in regulating cell growth, apoptosis, differentiation, and metabolism, and miRNAs are recognized as oncogenes or suppressor genes in tumors $[10,11]$. Recently, several investigations indicated that hypoxia is associated with the altered expression of miRNAs in RCC. For example, hypoxia could induce miR-30c downregulation, and $\mathrm{miR}-30 \mathrm{c}$ overexpression caused the inhibitory effects on 
epithelial-mesenchymal transition and cell migration in RCC cells [12]. Zhang and colleagues noted that miR-218 overexpressing under hypoxic conditions could suppress cell proliferation, inhibit the abilities of cell migration and invasion, but promote cell apoptosis by targeting CXCR7 downregulation in ACHN cells [13]. Xu et al. demonstrated that ccRCC cells under hypoxia could evoke the upregulation of has-miR-101 expression [14]. Recently, compelling evidence proved that NBAT1 suppressed the biological processes in renal carcinoma cells through the miR-346/GSK-3 $\beta$ axis [15]. However, the functional role of miR-346 under hypoxia in RCC remains unclear and needs further exploration.

$\mathrm{N}$-myc downstream-regulated gene 2 (NDRG2), a member of the recently found NDRG family participating in various malignancy tumors, has been proved to be a suppressor gene in cancers, which played a crucial role in regulating tumor growth [16]. Accumulative evidence demonstrated that NDRG2 could contribute to inhibit tumor biological processes such as cell migration, differentiation, and invasion, and enhance apoptosis [17]. Yang et al. reported that NDRG2 expression was downregulated in esophageal cancer tissues, while NDRG2 overexpression could block the cell cycle and inhibit cell invasion, migration, and EMT in TE-13 cells [18]. Additionally, NDRG2 silencing enhanced proliferation and invasion in RKO cells, which might provide a novel biomarker for colorectal cancer [19]. Moreover, a growing body of researches indicated that NDRG2 was involved in RCC. NDRG2 attenuated the renal carcinoma cell viability and triggered the G1 phase arrest in A-498 cells [20]. Liang et al. noted that NDRG2 might be a key factor, which was associated with the poor prognosis of clear cell RCC [21].

In the present study, we investigated the effects of hypoxia on RCC cell proliferation, migration, and invasion. Then we analyzed miR-346 expression in RCC cells treated with normoxia or hypoxia. Subsequently, the biological function effects of miR-346 on RCC cells were examined, and the downstream target gene expression was measured. This study might provide a new option for the diagnosis and treatment of RCC.

\section{Materials and methods}

Cell lines and cell culture. OS-RC- 2 and 786-O cell lines used in the present study were both human renal carcinoma cell lines, which were obtained from the Cell Resources Center of the Chinese Academy of Sciences (Shanghai, China). RPMI-1640 medium supplemented with $100 \mathrm{U} / \mathrm{ml}$ penicillin, $100 \mu \mathrm{g} / \mathrm{ml}$ streptomycin (Sigma-Aldrich, St. Louis, MO, USA) and $10 \%$ fetal bovine serum (Gibco-BRL, Gaithersburg, MD, USA). All cells were maintained at $37^{\circ} \mathrm{C}$ in a cell incubator with $5 \% \mathrm{CO}_{2}$ for normal oxygen. The hypoxic environment was established by setting the oxygen supply at $1 \%$.

Cell transfection. OS-RC-2 and 786-O cells were transfected with miR-346 inhibitor (in-miR-346), miR-346 mimic (miR-346), pcDNA3.1-NDRG2 (NDRG2), NDRG2 siRNA (si-NDRG2), and their corresponding scrambled controls in-miR-con, miR-con, pcDNA, si-con designed and purchased from GenePharma Co., Ltd. (Shanghai, China) by using Lipofectamine 2000 (Invitrogen, Carlsbad, CA, USA) in this study.

Cell proliferation assay. 3-(4,5-dimethyl-2-thiazolyl)2,5-diphenyl-2-H-tetrazolium bromide (MTT) assay was performed to evaluate cell proliferation. Cell viability was detected after cells treated with normoxia or hypoxia for $24 \mathrm{~h}, 48 \mathrm{~h}$, and $72 \mathrm{~h}$. Briefly, $20 \mu \mathrm{l}$ MTT was added into the incubation medium, and then the medium was replaced and $150 \mu \mathrm{l}$ DMSO per well was used to treat the cells. In the end, the absorbance was measured using a microplate reader (Bio-Rad Laboratories, Hercules, CA, USA) at $490 \mathrm{~nm}$.

Cell migration and invasion assays. Transwell assay (Bio-Rad Biosciences) was carried out to examine the migration and invasion viability of OS-RC-2 and 786-O cells. The transwell chambers were divided into two parts, including the upper chamber and lower chamber. Cells were incubated with the free medium in the upper chamber pre-coated with Matrigel for invasion, whereas the non-coated Matrigel upper chamber was used for migration. Meanwhile, the lower chamber was treated with RPMI-1640 plus 10\% FBS. Then, the cells that did not move were scraped using cotton tips. The cells on the bottom chamber were fixed using $10 \%$ formalin, stained with hematoxylin and counted under an inverted microscope.

Quantitative real-time PCR (qRT-PCR). Total RNA was isolated from human renal carcinoma cells exposed to hypoxia or normoxia using TRIzol reagent (Invitrogen). Then SYBR Premix Ex Taq II (Tli RNaseH PlusF) kit (Takara, Tokyo, Japan) was performed to detect the expression of miR-346 and NDRG2 in OS-RC-2 and 786-O cells. U6 and GAPDH were used as the internal reference for miRNA and the target gene detection, respectively. miR-346, (F) 5'-CACGGATCCCTTGTCAGCAAGGAGTG-3', (R) 5'-CGGAATTCTAGGTTGGGAGCGAAGTG-3'; U6, (F) 5'-CTCGCTTCGGCAGAC-3', (R) 5'-AACGCTTACGAATTT-3'; GAPDH, (F) 5'-GAPDH AAGAAGGTGGTGAAGCAGGC-3', (R) 5'-GTCAAAGGTGGAGGAGTGGG-3'; NDRG2, (F) 5'-CCCTGTGTTCCCTTTGGGAT-3', (R) 5'-GTGAGGCCTGTTAGCTTGTG-3'. The $2^{-\Delta \Delta C t}$ method was performed to analyze the expression of relative genes.

Dual-luciferase reporter assay. Wild-type NDRG2 3'UTR sequence that contained the predicted miR-346 binding sites and mutant NDRG2 3'UTR sequence without miR-346 binding sites (Ribobio, Shanghai, China) were inserted into the pGL3 vector (Promega, Madison, WI, USA), respectively. Luciferase activity was detected after cells co-transfected with miR-346 mimic or negative control (Beyotime Biotechnology Co. Ltd., Shanghai, China) and wild-type or mutant NDRG luciferase reporter vector using the Dual Luciferase Reporter $\left(D_{L R}^{\mathrm{TM}}\right)$ Assay System (Promega). 
RNA immunoprecipitation (RIP) assay. Magna RIP Kit (EMD Millipore Corporation, Darmstadt, Germany) was used to carry out the RIP assay. OS-RC-2 and 786-O cells were transfected with miR-346 mimic or control. RIP lysis buffer was used to lyse cells, and the lysates were collected to add the antibody against Ago2 (Abnova, Taiwan, China) or IgG (Abnova) conjugated with magnetic beads (EMD Millipore). The expression of NDRG2 was measured using qRT-PCR.

Western blot. Total protein was extracted from cells using a lysis buffer (Beyotime). Then a BCA protein assay kit (Beyotime) was used to quantify the concentration of the protein. Subsequently, $40 \mu \mathrm{g}$ protein were used for immunoblotting by SDS-PAGE. Then, proteins were transferred onto PVDF membranes. After block with 5\% non-fat dried milk for $2 \mathrm{~h}$, the membranes were incubated overnight at $4^{\circ} \mathrm{C}$ with primary antibodies, then washed by PBS, followed by the incubation with secondary antibodies. Primary antibodies against NDRG2 (1:1000; ab174850, Abcam, Cambridge, UK), $\beta$-actin (1:1000, ab179467, Abcam), and HRP conjugated secondary antibodies bought from Cell Signaling Technology (MA, USA) were used in the present research.

Statistical analysis. Data were analyzed by SPSS 18.0 software and showed as means \pm standard deviation (SD). Student's t-test was used to evaluate the difference between two comparison groups, and $\mathrm{p}<0.05$ means the statistically significant difference.

\section{Results}

Hypoxia promotes cell proliferation, migration, and invasion of RCC cells. To investigate the effects of hypoxia on renal carcinoma cells, we performed cell proliferation, migration, and invasion assays. We firstly detected the cell viability after cells cultured under normoxic or hypoxic conditions for $24,48,72 \mathrm{~h}$, the data indicated that cell proliferation was significantly enhanced when cells were treated with $1 \% \mathrm{O}_{2}$ for $48 \mathrm{~h}$ and $72 \mathrm{~h}$ compared with cells treated with normoxia (Figure 1A). Meanwhile, consistent with the result showed in cell proliferation assay, cell migration (Figure 1B), and invasion (Figure 1C) abilities were notably promoted by hypoxia both in OS-RC-2 and 786-O cells as relative to normoxia group.

Hypoxia upregulates miR-346 expression in RCC cells. OS-RC-2 and 786-O cells were cultured in a hypoxic $\left(1 \% \mathrm{O}_{2}\right)$ environment for $12,24,48$, and $72 \mathrm{~h}$ to investigate the expression of miR-346 in response to hypoxia. The data displayed that hypoxia remarkably upregulated miR-346 expression in a time-dependent manner in OS-RC-2 cells, as well as in 786-O cells (Figures 2A, 2B). These results demonstrated that miR-346 was sensitively responsive to hypoxia.

miR-346 downregulation attenuates hypoxia-evoked RCC cell proliferation, migration, and invasion. Moreover, to investigate the effects of miR-346 on regulating the progression of hypoxia-induced RCC cells, RCC cells under hypoxic conditions were transfected with miR-346 inhibitor and negative control. As indicated by qRT-PCR, miR-346 expression was suppressed by miR-346 inhibitor in OS-RC-2 (Figure $3 \mathrm{~A}$ ) and 786-O (Figure. 3B) cells. Meanwhile, the function of miR-346 on cell proliferation was detected by MTT assay. We found that cell proliferation was effectively enhanced by exposing RCC cells to hypoxia, whereas hypoxia-induced cell proliferation was distinctly restored by downregulating miR-346 (Figures 3C, 3D). Then, we evaluated the effects of miR-346 on cell migration and invasion induced by hypoxia. As described in Figures $3 \mathrm{E}$ and 3F, cell migration was induced by hypoxia in RCC cells. However, decrement of miR-346 weakened hypoxia-induced migration both in OS-RC-2 and 786-O cells. Consistent with the results of cell migration, transfection with miR-346 inhibitor also attenuated hypoxia-evoked cell invasion in RCC cells (Figures 3G, $3 \mathrm{H})$. We also detected the effects of the miR-346 inhibitor on proliferation, migration, and invasion of OS-RC-2 and 786-O cells in normoxia. As shown in Supplementary Figures S1A and $\mathrm{S} 1 \mathrm{~B}, \mathrm{miR}-346$ deletion could significantly inhibit the proliferative ability of OS-RC-2 and 786-O cells in normoxia. Consistently, the number of migrated and invaded OS-RC-2 and 786-O cells were dramatically decreased by downregulating miR-346 in normoxia (Supplementary Figures S1C, S1D). Taken together, knockdown of miR-346 could suppress cell growth and metastasis in OS-RC-2 and 786-O cells in normoxic conditions, and miR-346 deletion faded hypoxiainduced cell progression including proliferation, migration, and invasion.

NDRG2 is a target of miR-346. To explore the potential mechanism of miR-346 on regulating hypoxic RCC cells, a bioinformatics analysis by TargetScan was carried out to predict the potential target gene of miR-346 and discovered that NDRG2 contained the putative binding sites of miR-346, as displayed in Figure 4A. Subsequently, dual luciferase assays were performed. The data demonstrated that luciferase activities were significantly decreased by miR-346 overexpression in RCC cells transfected with wild-type NDRG2, while the luciferase activities were not changed in NDRG2-MUT RCC cells (Figures 4B, 4C). Furthermore, the RIP assay was carried out. The results suggested that NDRG2 was enriched by the anti-AGO2 antibody as relative to the negative control group (IgG) (Figures 4D, 4E). Moreover, transfection of miR-346 mimic led to the depression of NDRG2 expression, whereas the expression of NDRG2 was increased by miR-346 knockdown in RCC cells (Figures 4F, 4G). Considering all these data, NDRG2 was a direct target of miR-346 and negatively regulated by miR-346.

NDRG2 overexpression alleviates proliferation, migration, and invasion of RCC cells under hypoxia. Then, we explored the functional role of NDRG2 in hypoxia-induced RCC cells. qRT-PCR data indicated that NDRG2 mRNA expression was significantly suppressed by hypoxia in OS-RC-2 and 786-O cells (Figures 5A, 5B). Before functional assays, western blot assay illustrated that the transfection 
A
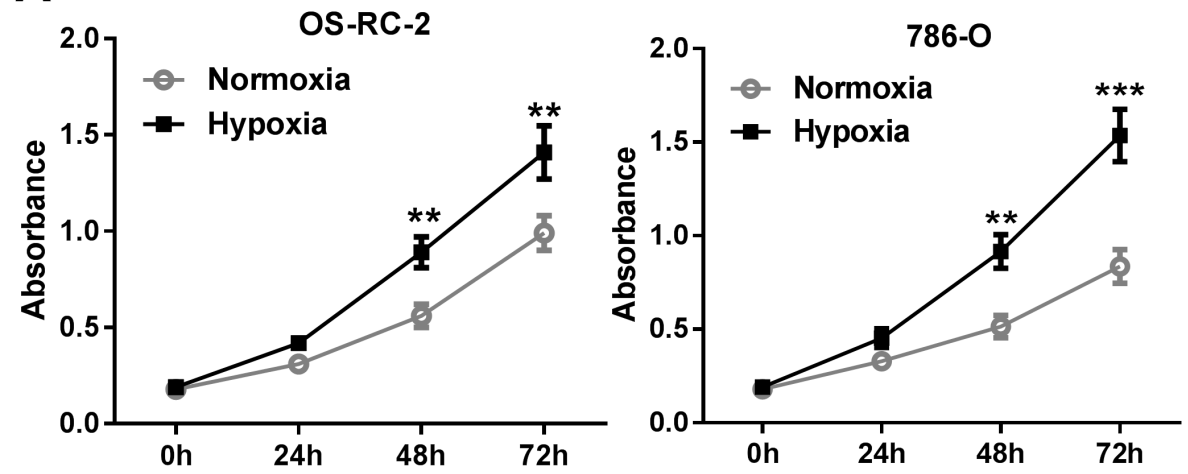

B

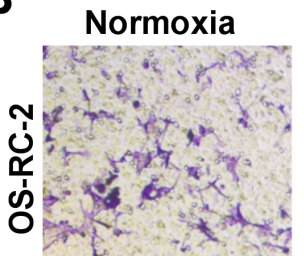

Normoxia

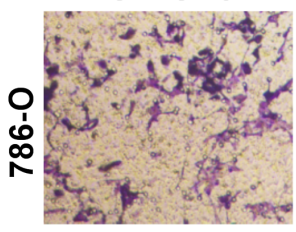

C

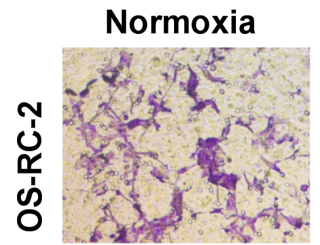

Normoxia

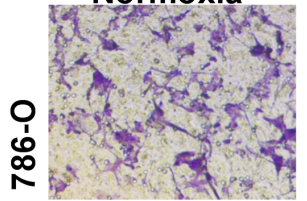

Hypoxia

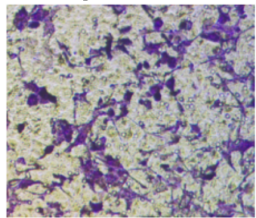

Hypoxia
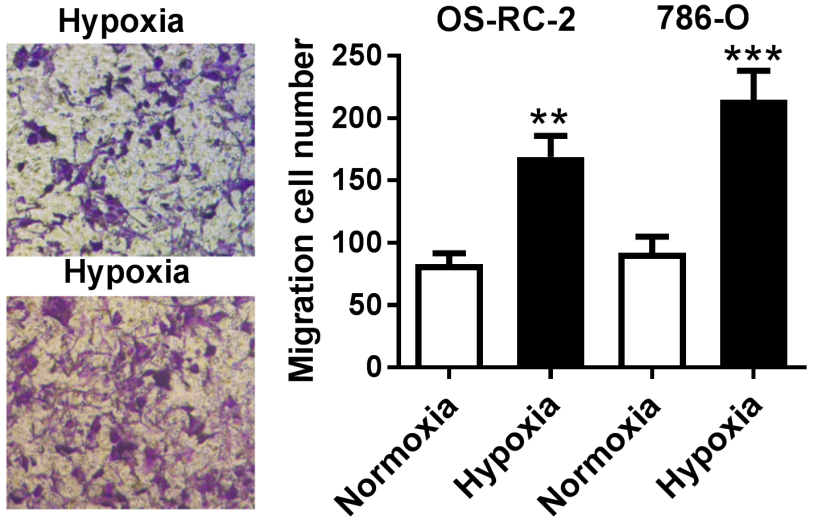

OS-RC-2 786-O

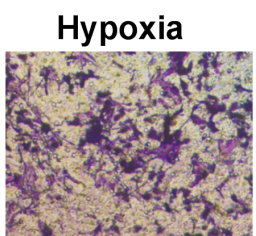

Hypoxia
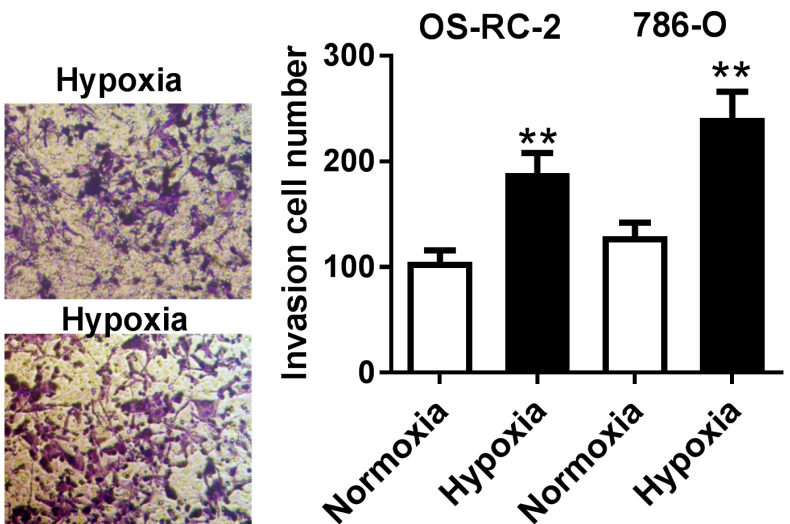

Figure 1. Hypoxia promotes cell proliferation, migration, and invasion of RCC cells. A) MTT assays were conducted to detect cell proliferation of OS-RC-2 and 786-O cells inducted with normoxia or hypoxia for 24,48 , and $72 \mathrm{~h}$. B) Transwell migration and C) invasion assays were performed to measure the migratory and invasive ability of OS-RC-2 and 786-O cells cultured under normoxic or hypoxic conditions. ${ }^{* *} \mathrm{p}<0.01,{ }^{* * *} \mathrm{p}<0.001$

of pcDNA-NDRG2 significantly increased NDRG2 expression (Figures 6A, 6B). As represented in Figures 6C and 6D, NDRG2 overexpression remarkably suppressed cell proliferation induced by hypoxia in RCC cells. Then, we investigated whether NDRG2 can regulate migration and invasion of RCC cells triggered by hypoxia. The data indicated that NDRG2 overexpressing could attenuate cell migration induced by hypoxia in OS-RC-2 and 786-O cells (Figures 6E,
6F). Meanwhile, consistent with the results of migration assays, we found that cell invasion in hypoxia evoked RCC cells were inhibited by NDRG2 upregulation (Figures 6G, $6 \mathrm{H})$. Overall, we concluded that NDRG2 overexpression suppressed hypoxia-triggered proliferation, migration, and invasion in RCC cells.

NDRG2 knockdown attenuates the inhibitory effects of miR-346 on proliferation, migration, and invasion in 

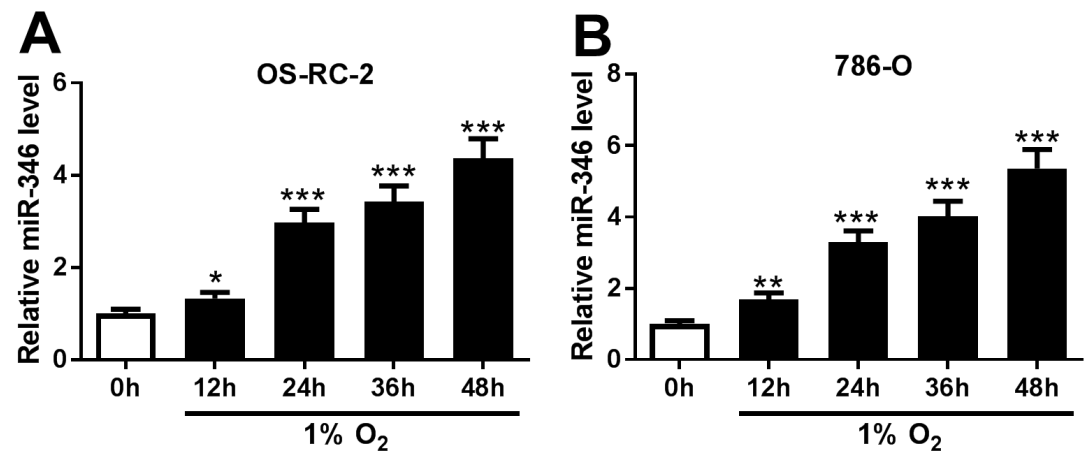

Figure 2. Hypoxia upregulates miR-346 expression in RCC cells. A, B) miR-346 expression was measured by qRT-PCR in OS-RC-2 and 786-O cells treated with $1 \% \mathrm{O}_{2}$ for $12,24,36,48 \mathrm{~h} .{ }^{*} \mathrm{p}<0.05,{ }^{* *} \mathrm{p}<0.01,{ }^{* * *} \mathrm{p}<0.001$

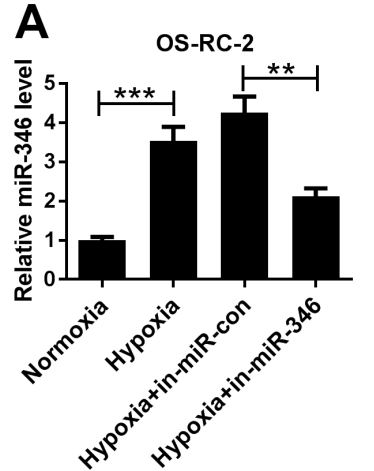

E

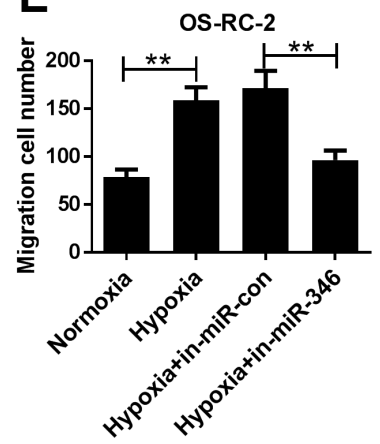

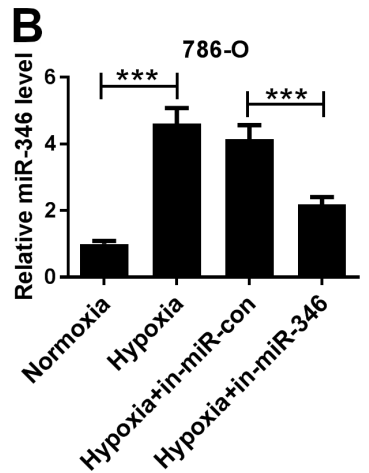

$\mathbf{F}$

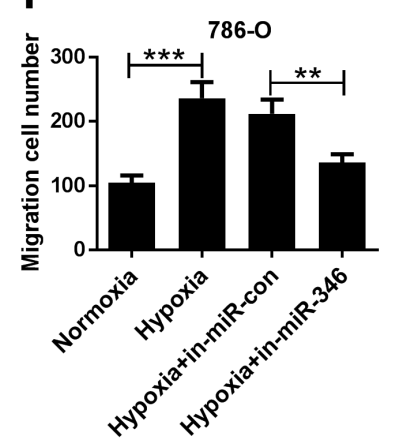

C

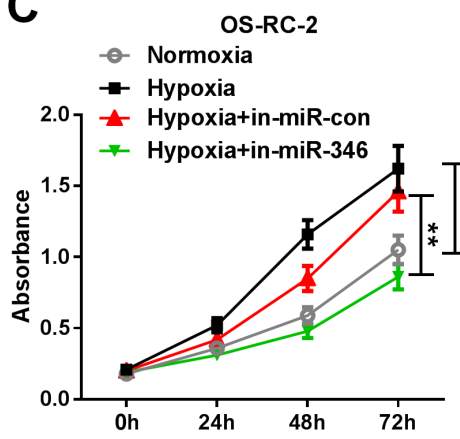

G

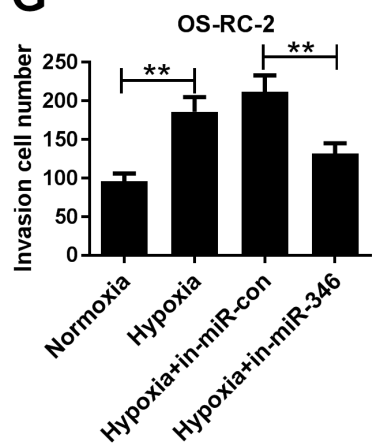

D

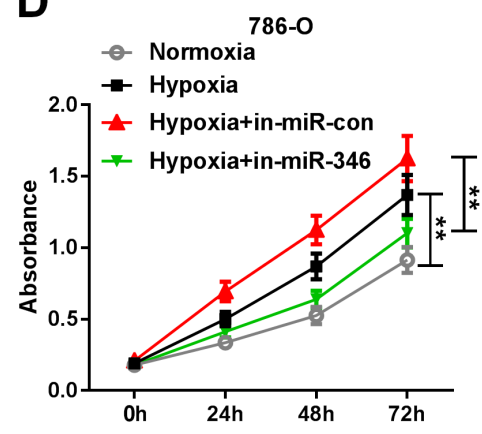

H

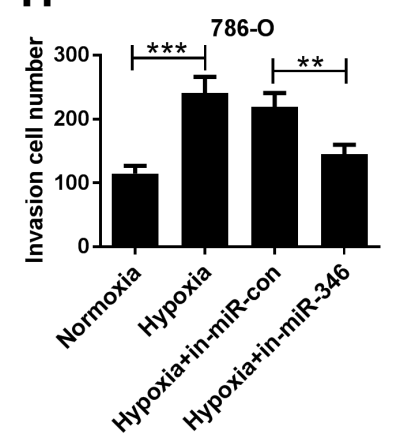

Figure 3. miR-346 downregulation attenuates hypoxia-evoked RCC cell proliferation, migration, and invasion. A, B) miR-346 expression in OS-RC-2 and 786-O cells was detected using western blot assay after transfection with miR-346 inhibitor or negative control, followed by cells cultured with normoxia or hypoxia. C, D) Proliferation of OS-RC-2 and 786-O cells transfected with miR-346 inhibitor or negative control, and exposed to normoxic or hypoxic environment for $24,48,72 \mathrm{~h}$, was examined by MTT assay. E, F) Migration cell number was measured by transwell migration assay in OSRC-2 and 786-O cells transfected with miR-346 inhibitor or negative control, followed by the induction of normoxia or hypoxia. G, H) Invasion cell number was evaluated by transwell invasion assay. ${ }^{* *} \mathrm{p}<0.01,{ }^{* * *} \mathrm{p}<0.001$

hypoxia-cultured RCC cells. Finally, rescue assays were performed to explore the roles of miR-346 and NDRG2 in RCC cells under hypoxia. RCC cells used in rescue assays were cultured under hypoxic conditions. Western blot data illustrated that the transfection of miR-346 inhibitor upregulated the expression of NDRG2, whereas the upregulation of NDRG2 induced by miR-346 inhibitor was reversed by NDRG2 knockdown (Figures 7A, 7B). As shown in
Figures 7C and 7D, compared with the miR-346 inhibitor group, NDRG2 silencing significantly enhanced proliferation and reversed the inhibitory effect of miR-346 on cell proliferation. We also found that NDRG2 silencing reversed the inhibitory effects of the miR-346 inhibitor on migration in OS-RC-2 and 786-O cells treated with $1 \% \mathrm{O}_{2}$ (Figures 7E, $7 F)$. Rescue assays further indicated that NDRG2 deletion partially reversed the invasion of OS-RC-2 and 786-O cells in 
comparison to RCC cells transfected with miR-346 inhibitor (Figures 7G, 7H). Collectively, these results demonstrated that NDRG2 deletion resisted the effects of the miR-346 inhibitor on proliferation, migration, and invasion of RCC cells under hypoxic conditions.

\section{Discussion}

Hypoxia, which represents a low oxygen environment, has been proved to be a factor in solid tumors prognosis, including RCC. Tumor hypoxia may result in the changes of phenotypes to adapt to the hypoxic conditions and induce cell growth, metastasis, and invasion [22]. For example, Yang and colleagues found that gastric cancer cell viability and migration activity were increased by hypoxia [23]. Furthermore, smooth muscle cells treated with $1 \% \mathrm{O}_{2}$ triggered proliferation upregulation as relative to $21 \% \mathrm{O}_{2}$ [24]. Recently research indicated that hypoxia could evoke kidney damage in rats, accompanying the activation of HIF-1a [25]. HIF-1 $\alpha$ and HIF-2a played crucial roles in cell growth and metastasis in tumors. Two VHL-defective RCC cell lines OS-RC-2 and 786-O cells were used in the present research. OS-RC2 cells were confirmed to express the HIF-1 $\alpha$ and HIF-2a, while 786-O expressed HIF-2 $\alpha$ alone. VEGF and GLUT1 are confirmed to be HIF-responsive genes, which were important regulators in cell growth and metastasis, and the findings from the previous studies indicated that VEGF and GLUT1 could be regulated by HIF- $2 \alpha$ but not HIF-1 $\alpha$ in VHL-defec-

A Position on chromosome: 14:21490016-21490022 of
NDRG2 3'UTR

NDRG2-WT 5'...AUCUCUGGACCAGCUUGCAGAC A...3' miR-346 3'UCUCCGUCCGUACGCCCGUCUGU 5 NDRG2-MUT 5'...AUCUCUGGACCAGCUUUACUGAG...3'
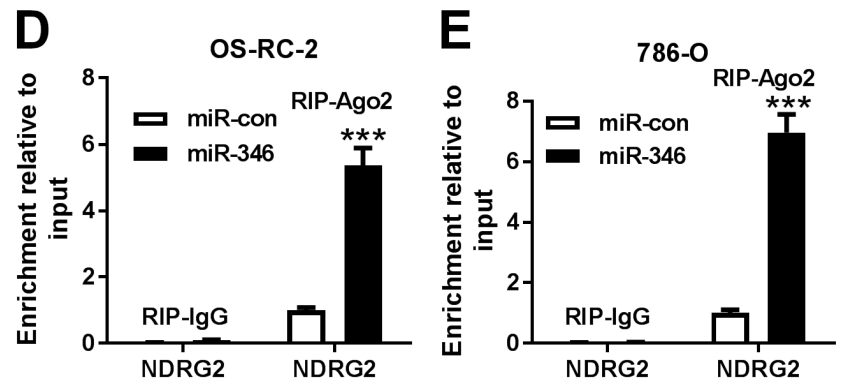

B
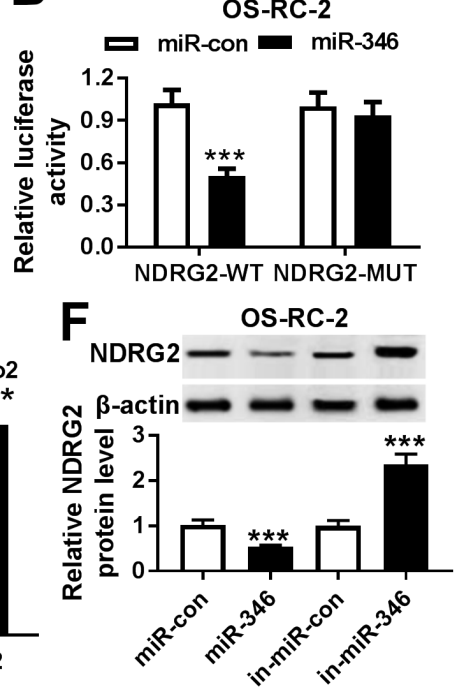

C
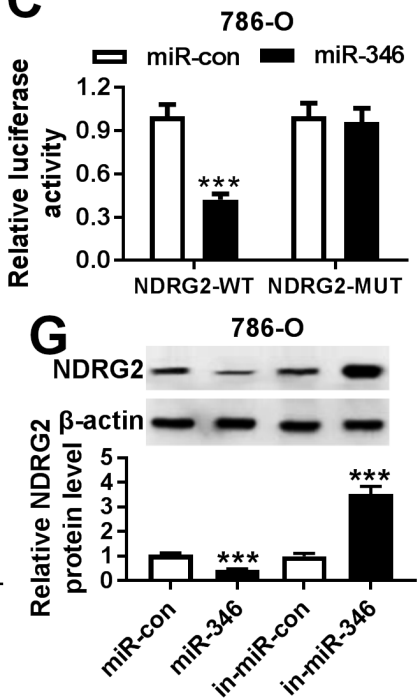

Figure 4. NDRG2 is a target of miR-346. A) MicroT-CDS predicted that the NDRG2 mRNA 3'UTR sequence contained the binding sites of miR-346. B, C) Dual-Luciferase reporter assay was carried out to detect the luciferase activity in co-transfection with miR-346 mimic or miR-con and NDRG2WT or NDRG2-MUT OS-RC-2 and 786-O cells. D, E) The level of NDRG2 enriched by RIP-Ago2 was assessed in OS-RC-2 and 786-O cells transfected with miR-346 mimic or miR-con by qRT-PCR. F, G) NDRG2 protein expression levels in OS-RC-2 and 786-O cells transfected with miR-346 mimic or inhibitor, and their corresponding negative controls were detected by western blot. ${ }^{* * *} \mathrm{p}<0.001$
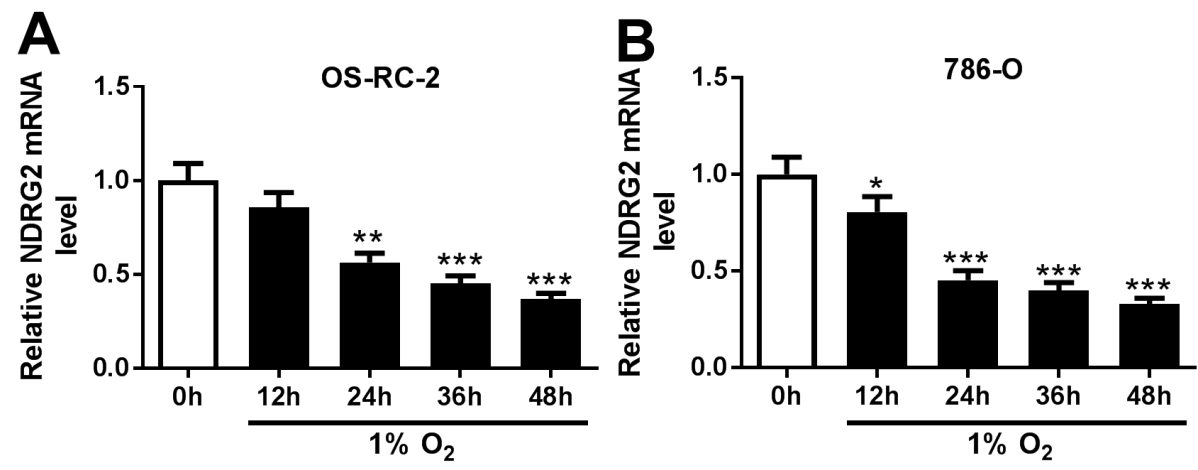

Figure 5. NDRG2 expression was downregulated by hypoxia. A, B) NDRG2 expression w in OS-RC-2 and 786-O cells treated with $1 \%$ $\mathrm{O}_{2}$ for $12,24,36$, $48 \mathrm{~h}$ was measured by qRT-PCR. ${ }^{*} \mathrm{p}<0.05,{ }^{* *} \mathrm{p}<0.01,{ }^{* *} \mathrm{p}<0.001$ 


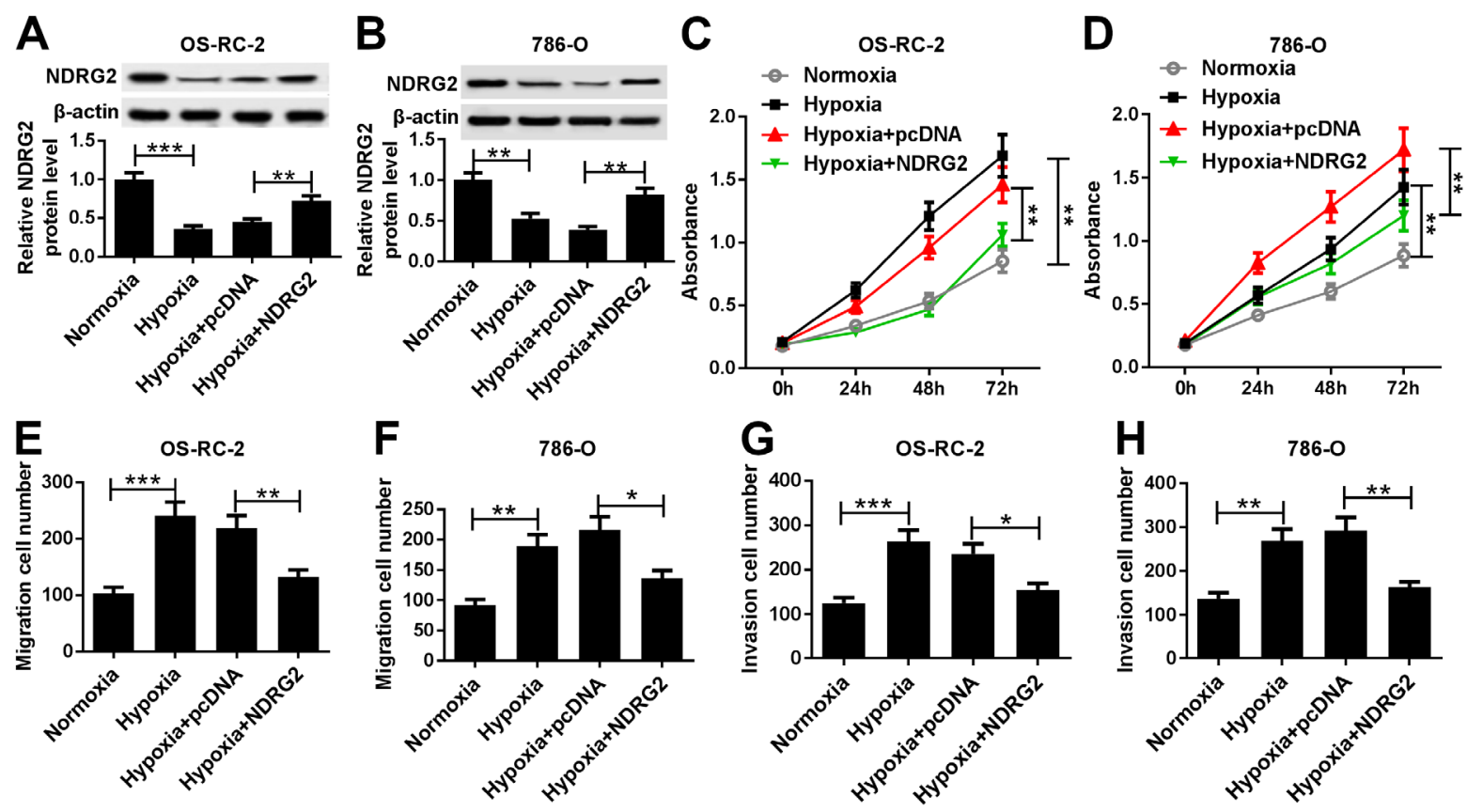

Figure 6. NDRG2 overexpression alleviates proliferation, migration, and invasion induced by hypoxia in RCC cells. OS-RC-2 and 786-O cells were transfected with pcDNA or pcDNA-NDRG2 prior to exposure to a normoxic or hypoxic environment. A, B) NDRG2 expression was measured by western blot assay. C, D) Cell proliferation was measured by MTT assay. E, F) Migration cell number was detected by transwell migration assay. G, H) Invasion cell number was evaluated by transwell invasion assay. ${ }^{*} \mathrm{p}<0.05,{ }^{* *} \mathrm{p}<0.01,{ }^{* * *} \mathrm{p}<0.001$

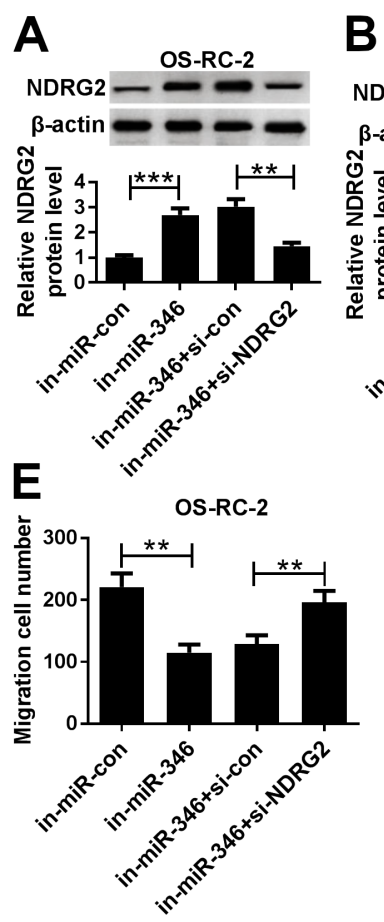

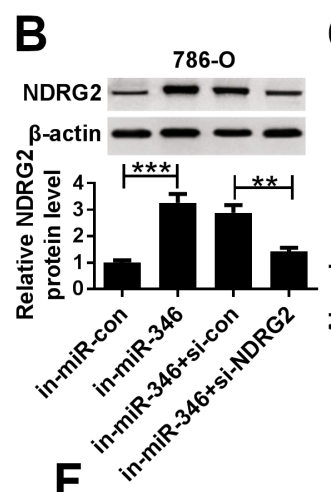

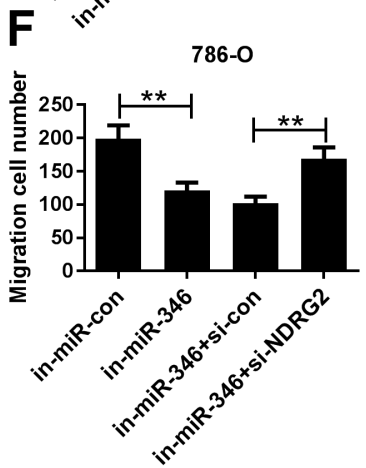

C

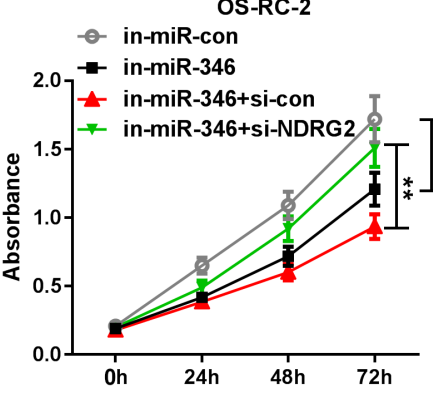

G

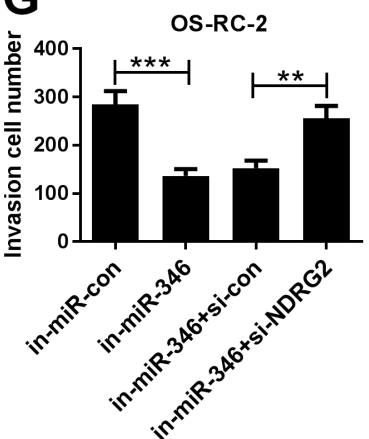

D

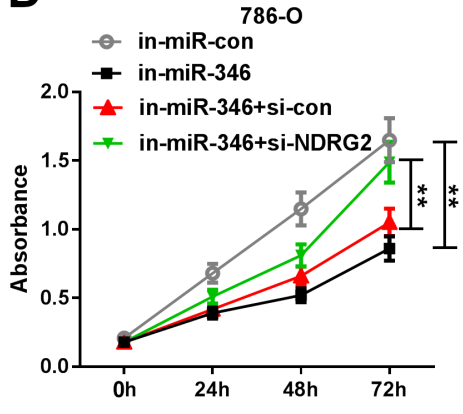

H

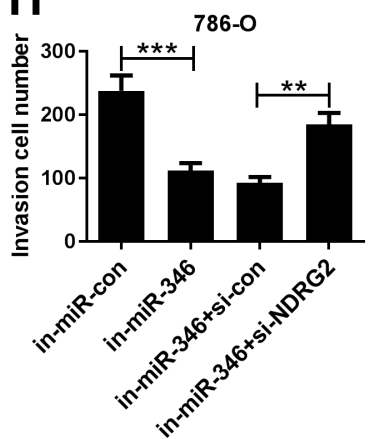

Figure 7. NDRG2 knockdown attenuates the inhibitory effects of miR-346 on proliferation, migration, and invasion in hypoxia-cultured RCC cells. OS-RC-2 and 786-O cells cultured in hypoxia were transfected with miR-346 inhibitor or miR-con and si-NDRG2 or si-con. A, B) NDRG2 expression was measured by western blot assay. C, D) Cell proliferation was examined by MTT assay, followed by treatment in hypoxic conditions for 24, 48, and $72 \mathrm{~h}$. E, F) The migration ability was evaluated by transwell migration assay. G, H) Invasion cell number was evaluated by transwell invasion assay. ${ }^{* *} \mathrm{p}<0.01,{ }^{* *} \mathrm{p}<0.001$ 
tive RCC cells [26]. In our study, we discovered that hypoxia could promote proliferation, migration, and invasion of OS-RC-2 and 786-O cells compared with cells treated with normal oxygen. The results were consistent with the findings from Zhang and colleagues that the migration and invasion cell numbers of renal cell carcinoma cells were increased by treating with hypoxia [13]. Our results indicated that HIF-2a might be a regulator factor in the progression of RCC in hypoxia.

Hypoxia-evoked dysregulated expression of miRNAs has been demonstrated $[27,28]$. The latest researches suggested that miR-346 was closely associated with tumor pathological process development and exerted an oncogenic role in cell apoptosis, migration, and proliferation [29, 30]. For example, miR-346 expression was upregulated in nasopharyngeal carcinoma tissues, and miR-346 overexpression enhanced nasopharyngeal carcinoma cell migration and invasion [31]. Yang et al. found that the biological functions including colony formation, proliferation, and migration of MCF-7 cells were promoted by miR-346, whereas miR-346 could decrease cell apoptosis in breast cancer [32]. Sun and colleagues indicated that miR-346 was discovered to be increased in hepatocellular carcinoma cells and induce cell growth and migration in hepatocellular carcinoma through suppressing KLF14 [33]. Of note, Xue et al. found that miR-346 was involved in RCC pathological process [15]. In our study, we examined the expression of miR-346 in RCC cells cultured with hypoxia and discovered that miR-346 was significantly increased in RCC cells under hypoxia. Subsequently, we investigated the functional role of miR-346 in RCC cells under hypoxic environment. The results showed that the downregulation of miR-346 by expressing miR-346 inhibitor could depress cell proliferation and decrease migration and invasion cell numbers under hypoxia.

The regulation of miRNAs on cell apoptosis, proliferation, and metastasis in tumors through regulating the downstream gene expression has been widely reported. In the present research, we found that NDRG2 was a downstream target gene of miR-346 and confirmed that miR-346 negatively regulated NDRG2 expression in RCC cells. Notably, NDRG2 has been proved to be a risk factor, which can suppress pathological tumor processes in diverse human cancers [34]. Additionally, NDRG2 has been recognized as a stress-responsive gene that can respond to hypoxia in cellular stress [35]. The previous work indicated that NDRG2 suppressed the proliferation of renal cell carcinoma cell lines [36]. Ma et al. reported that NDRG2 could inhibit clear cell renal cell carcinoma invasion by regulating MMP-9 activity [37]. In this study, we found that the overexpression of NDRG2 led to inhibitory effects on cell proliferation, migration, and invasion induced in RCC cells under hypoxia. Meanwhile, we also observed that NDRG2 deletion partially reversed the suppression of miR-346 inhibitor on proliferation, migration, and invasion in RCC cells cultured in hypoxic conditions.
Taken together, the present study confirmed that hypoxia promoted the development of tumor growth in RCC. And miR-346 was highly expressed, whereas NDRG2 was lowly expressed in RCC cells treated with hypoxia. Our observation indicated that miR-346 significantly accelerated proliferation, migration, and invasion of renal cell carcinoma cells under hypoxic conditions by targeting NDRG2.

Supplementary information is available in the online version of the paper.

Acknowledgments: The project was supported by Zhanjiang City Financial Special Reserve Fund Project (grant no. 2016C01019).

\section{References}

[1] HOLLINGSWORTH JM, MILLER DC, DAIGNAULT S, HOLLENBECK BK. Rising incidence of small renal masses: a need to reassess treatment effect. J Natl Cancer Inst 2006; 98: 1331-1334. https://doi.org/10.1093/jnci/djj362

[2] SIEGEL RL, MILLER KD, JEMAL A. Cancer statistics, 2015. CA Cancer J Clin 2015; 65: 5-29. https://doi. org/10.3322/caac. 21254

[3] CAPOGROSSO P, CAPITANIO U, LA CROCE G, NINI A, SALONIA A et al. Follow-up After Treatment for Renal Cell Carcinoma: The Evidence Beyond the Guidelines. European urology focus 2016; 1: 272-281. https://doi.org/10.1016/j. euf.2015.04.001

[4] THOMAS JS, KABBINAVAR F. Metastatic clear cell renal cell carcinoma: A review of current therapies and novel immunotherapies. Crit Rev Oncol Hematol 2015; 96: 527-533. https://doi.org/10.1016/j.critrevonc.2015.07.009

[5] NISHIKIMI T, TSUZUKI T, FUJITA T, SASSA N, FUKATSU A et al. The post-operative pathological prognostic parameters of clear cell renal cell carcinoma in pT1a cases. Pathol Int 2011; 61: 116-121. https://doi.org/10.1111/j.14401827.2010.02633.x

[6] POUYSSEGUR J, DAYAN F, MAZURE NM. Hypoxia signalling in cancer and approaches to enforce tumour regression. Nature 2006; 441: 437-443. https://doi.org/10.1038/ nature 04871

[7] KONG HS, LEE S, BEEBE K, SCROGGINS B, GUPTA G et al. Emetine promotes von Hippel-Lindau-independent degradation of hypoxia-inducible factor-2alpha in clear cell renal carcinoma. Mol Pharmacol 2010; 78: 1072-1078. https:// doi.org/10.1124/mol.110.066514

[8] SUN J, JIANG Z, LI Y, WANG K, CHEN X et al. Downregulation of miR-21 inhibits the malignant phenotype of pancreatic cancer cells by targeting VHL. Onco Targets Ther 2019; 12: 7215-7226. https://doi.org/10.2147/OTT.S211535

[9] MO YY. MicroRNA regulatory networks and human disease. Cellular and molecular life sciences : CMLS 2012; 69: 35293531. https://doi.org/10.1007/s00018-012-1123-1

[10] FARH KK, GRIMSON A, JAN C, LEWIS BP, JOHNSTON WK et al. The widespread impact of mammalian MicroRNAs on mRNA repression and evolution. Science 2005; 310: 1817-1821. https://doi.org/10.1126/science.1121158 
[11] KENT OA, MENDELL JT. A small piece in the cancer puzzle: microRNAs as tumor suppressors and oncogenes. Oncogene 2006; 25: 6188-6196. https://doi.org/10.1038/sj.onc.1209913

[12] HUANG J, YAO X, ZHANG J, DONG B, CHEN Q et al. Hypoxia-induced downregulation of miR-30c promotes epithelial-mesenchymal transition in human renal cell carcinoma. Cancer Sci 2013; 104: 1609-1617. https://doi.org/10.1111/ cas. 12291

[13] ZHANG S, HONG Z, LI Q, LEI J, HUANG H et al. Effect of MicroRNA-218 on the viability, apoptosis and invasion of renal cell carcinoma cells under hypoxia by targeted downregulation of CXCR7 expression. Biomed Pharmacother 2016; 80: 213-219. https://doi.org/10.1016/j.biopha.2016.03.011

[14] XU X, LIU C, BAO J. Hypoxia-induced hsa-miR-101 promotes glycolysis by targeting TIGAR mRNA in clear cell renal cell carcinoma. Mol Med Rep 2017; 15: 1373-1378. https://doi.org/10.3892/mmr.2017.6139

[15] XUE S, WANG S, LI J, GUAN H, JIANG S et al. LncRNA NBAT1 suppresses cell proliferation and migration via miR346/GSK-3beta axis in renal carcinoma. IUBMB life 2019; https://doi.org/10.1002/iub.2111

[16] MELOTTE V, QU X, ONGENAERT M, VAN CRIEKINGE W, DE BRUINE AP et al. The N-myc downstream regulated gene (NDRG) family: diverse functions, multiple applications. FASEB J 2010; 24: 4153-4166. https://doi.org/10.1096/ f. $09-151464$

[17] SHI H, LI N, LI S, CHEN C, WANG W et al. Expression of NDRG2 in esophageal squamous cell carcinoma. Cancer Sci 2010; 101: 1292-1299. https://doi.org/10.1111/j.13497006.2010.01529.x

[18] YANG CL, ZHENG XL, YE K, GE H, SUN YN et al. NDRG2 suppresses proliferation, migration, invasion and epithelialmesenchymal transition of esophageal cancer cells through regulating the AKT/XIAP signaling pathway. Int J Biochem Cell Biol 2018; 99: 43-51. https://doi.org/10.1016/j.biocel.2018.03.003

[19] HONG SN, KIM SJ, KIM ER, CHANG DK, KIM YH. Epigenetic silencing of NDRG2 promotes colorectal cancer proliferation and invasion. J Gastroenterol Hepatol 2016; 31: 164-171. https://doi.org/10.1111/jgh.13068

[20] MA JJ, LIAO CG, JIANG X, ZHAO HD, YAO LB et al. NDRG2 suppresses the proliferation of clear cell renal cell carcinoma cell A-498. J Exp Clin Cancer Res 2010; 29: 103. https://doi.org/10.1186/1756-9966-29-103

[21] LIANG ZL, KANG K, YOON S, HUANG SM, LIM JS et al. NDRG2 is involved in the oncogenic properties of renal cell carcinoma and its loss is a novel independent poor prognostic factor after nephrectomy. Ann Surg Oncol 2012; 19: 2763-2772. https://doi.org/10.1245/s10434-011-2204-3

[22] SEMENZA GL. Hypoxia-inducible factor 1: master regulator of O2 homeostasis. Curr Opin Genet Dev 1998; 8: 588-594. https://doi.org/10.1016/s0959-437x(98)80016-6

[23] YANG L, ZHANG W, WANG Y, ZOU T, ZHANG B et al. Hypoxia-induced miR-214 expression promotes tumour cell proliferation and migration by enhancing the Warburg effect in gastric carcinoma cells. Cancer Lett 2018; 414: 44-56. https://doi.org/10.1016/j.canlet.2017.11.007
[24] CHEN B, NELIN VE, LOCY ML, JIN Y, TIPPLE TE. Thioredoxin-1 mediates hypoxia-induced pulmonary artery smooth muscle cell proliferation. Am J Physiol Lung Cell Mol Physiol 2013; 305: L389-395. https://doi.org/10.1152/ ajplung.00432.2012

[25] LIU LL, LI D, HE YL, ZHOU YZ, GONG SH et al. miR-210 protects renal cell against hypoxia-induced apoptosis by targeting HIF-1 alpha. Mol Med 2017; 23: 258-271. https://doi. org/10.2119/molmed.2017.00013

[26] RAVAL RR, LAU KW, TRAN MG, SOWTER HM, MANDRIOTA SJ et al. Contrasting properties of hypoxiainducible factor 1 (HIF-1) and HIF-2 in von Hippel-Lindau-associated renal cell carcinoma. Mol Cell Biol 2005; 25: 5675-5686. https://doi.org/10.1128/MCB.25.13.56755686.2005

[27] ZHAO ZB, CHEN F, BAI XF. Long Noncoding RNA MALAT1 Regulates Hepatocellular Carcinoma Growth Under Hypoxia via Sponging MicroRNA-200a. Yonsei Med J 2019; 60: 727-734. https://doi.org/10.3349/ymj.2019.60.8.727

[28] BRAGA EA, FRIDMAN MV, LOGINOV VI, DMITRIEV AA, MOROZOV SG. Molecular Mechanisms in Clear Cell Renal Cell Carcinoma: Role of miRNAs and Hypermethylated miRNA Genes in Crucial Oncogenic Pathways and Processes. Front Genet 2019; 10: 320. https://doi.org/10.3389/ fgene.2019.00320

[29] GUO Z, LI J, SUN J, SUN L, ZHOU Y et al. miR-346 Promotes HCC Progression by Suppressing Breast Cancer Metastasis Suppressor 1 Expression. Oncol Res 2018; 26: 10731081. https://doi.org/10.3727/096504017X15145088802439

[30] YU Q, YANG X, DUAN W, LI C, LUO Y et al. miRNA-346 promotes proliferation, migration and invasion in liver cancer. Oncol Lett 2017; 14: 3255-3260. https://doi.org/10.3892/ ol.2017.6561

[31] YAN HL, LI L, LI SJ, ZHANG HS, XU W. miR-346 promotes migration and invasion of nasopharyngeal carcinoma cells via targeting BRMS1. J Biochem Mol Toxicol 2016; 30: 602607. https://doi.org/10.1002/jbt.21827

[32] YANG F, LUO LJ, ZHANG L, WANG DD, YANG SJ et al. MiR-346 promotes the biological function of breast cancer cells by targeting SRCIN1 and reduces chemosensitivity to docetaxel. Gene 2017; 600: 21-28. https://doi.org/10.1016/j. gene.2016.11.037

[33] WANG YG, LIU J, SHI M, CHEN FX. LncRNA DGCR5 represses the development of hepatocellular carcinoma by targeting the miR-346/KLF14 axis. J Cell Physiol 2018; 234: 572-580. https://doi.org/10.1002/jcp.26779

[34] HU W, YANG Y, FAN C, MA Z, DENG C et al. Clinical and pathological significance of N-Myc downstream-regulated gene 2 (NDRG2) in diverse human cancers. Apoptosis 2016; 21: 675-682. https://doi.org/10.1007/s10495-016-1244-3

[35] ARAYA-CALLIS C, HIEMKE C, ABUMARIA N, FLUGGE G. Chronic psychosocial stress and citalopram modulate the expression of the glial proteins GFAP and NDRG2 in the hippocampus. Psychopharmacology (Berl) 2012; 224: 209-222. https://doi.org/10.1007/s00213-012-2741-X 
[36] WU Z, LIAO H, SU J, YANG L, CHI Z et al. Adenovirusmediated NDRG2 inhibits the proliferation of human renal cell carcinoma cell line OS-RC-2 in vitro. Cell Biochem Biophys 2014; 70: 593-600. https://doi.org/10.1007/s12013-0149961-0
[37] MA JJ, KONG LM, LIAO CG, JIANG X, WANG Y et al. Suppression of MMP-9 activity by NDRG2 expression inhibits clear cell renal cell carcinoma invasion. Med Oncol 2012; 29: 3306-3313. https://doi.org/10.1007/s12032-012-0265-1 